\title{
Diagnostic performance of FDG-PET/MRI and WB-DW-MRI in the evaluation of lymphoma: a prospective comparison to standard FDG-PET/CT
}

Ken Herrmann 1,2, Marcelo Queiroz ${ }^{1}$, Martin W. Huellner ${ }^{1,3,6}$, Felipe de Galiza Barbosa ${ }^{1}$, Andreas Buck², Niklaus Schaefer ${ }^{1,5,6}$, Paul Stolzman ${ }^{1,3,6}$ and Patrick Veit-Haibach ${ }^{1,4,6^{*}}$

\begin{abstract}
Background: Use of FDG-PET/CT for staging and restaging of lymphoma patients is widely incorporated into current practice guidelines. Our aim was to prospectively evaluate the diagnostic performance of FDG-PET/MRI and WB-DW-MRI compared with FDG-FDG-PET/CT using a tri-modality PET/CT-MRI system.
\end{abstract}

Methods: From 04/12 to 01/14, a total of 82 FDG-PET/CT examinations including an additional scientific MRI on a tri-modality setup were performed in 61 patients. FDG-PET/CT, FDG-PET/MRI, and WB-DW-MRI were independently analyzed. A lesion with a mean ADC below a threshold of $1.2 \times 10^{-3} \mathrm{~mm}^{2} / \mathrm{s}$ was defined as positive for restricted diffusion. FDG-PET/CT and FDG-PET/MRI were evaluated for the detection of lesions corresponding to lymphoma manifestations according to the German Hodgkin Study Group. Imaging findings were validated by biopsy $(n=21)$, by follow-up imaging comprising CT, FDG-PET/CT, and/or FDG-PET/MRI $(n=32)$, or clinically $(n=25)$ (mean followup: 9.1 months).

Results: FDG-PET/MRI and FDG-PET/CT accurately detected 188 lesions in 27 patients. Another 54 examinations in 35 patients were negative. WB-DW-MRI detected 524 lesions, of which 125 (66.5\% of the aforementioned 188 lesions) were true positive. Among the 188 lesions positive for lymphoma, FDG-PET/MRI detected all 170 instances of nodal disease and also all 18 extranodal lymphoma manifestations; by comparison, WB-DW-MRI characterized 115 (67.6\%) and 10 (55.6\%) lesions as positive for nodal and extranodal disease, respectively. FDG-PET/MRI was superior to WB-DW-MRI in detecting lymphoma manifestations in patients included for staging (113 vs. 73), for restaging (75 vs. 52), for evaluation of high- (127 vs. 81) and low-grade lymphomas (61 vs. 46), and for definition of Ann Arbor stage (WB-DW-MRI resulted in upstaging in 60 cases, including 45 patients free of disease, and downstaging in 4).

Conclusion: Our results indicate that FDG-PET/CT and FDG-PET/MRI probably have a similar performance in the clinical work-up of lymphomas. The performance of WB-DW-MRI was generally inferior to that of both FDG-PET-based methods but the technique might be used in specific scenarios, e.g., in low-grade lymphomas and during surveillance.

Keywords: Whole-body, WB-DW-MRI, FDG, FDG-PET/CT, FDG-PET/MRI, Lymphoma

\footnotetext{
*Correspondence: Patrick.Veit-Haibach@usz.ch

'Department of Nuclear Medicine, University Hospital Zurich, Rämistrasse

100, CH-8091 Zurich, Switzerland

${ }^{4}$ Department of Diagnostic and Interventional Radiology, University Hospital

Zurich, Rämistrasse 100, CH-8091 Zurich, Switzerland

Full list of author information is available at the end of the article
} 


\section{Background}

Use of fluorodeoxyglucose positron emission tomography/computed tomography (FDG-PET/CT) for staging and restaging of lymphoma patients is now clinical routine and is widely incorporated into current practice guidelines [1]. Recent advances in magnetic resonance imaging (MRI) technology and MRI sequences have led to the introduction of whole-body diffusion-weighted MRI (WB-DW-MRI) and allowed for calculation of apparent diffusion coefficients (ADC) [2]. WB-DW-MRI is expected to improve staging accuracy due to the potential improvement in lesion-to-background contrast [3]. Previously published studies comparing WB-DW-MRI and FDG-PET/CT reported kappa values for method agreement ranging from 0.51 to $0.85[4,5]$. The fact that all major vendors offer hybrid scanners combining MRI, PET, and/or CT technology allows for direct comparison of single imaging modalities as well as hybrid approaches [6].

Potential advantages of WB-DW-MRI in comparison with either FDG-PET/CT or FDG-PET/MRI include no radiation burden, the possibility of protocol standardization, and high tumor-to-background contrast; in addition, image acquisition times are comparable.

Promising initial results encouraged authors to advocate WB-DW-MRI as a potential replacement for FDG-PET/ CT [7]. However, as yet no prospectively validated ADC criteria have been established for differentiation of lymphomatous from non-lymphomatous lymph nodes when using WB-DW-MRI. Moreover, few data are currently available regarding the performance of FDG-PET/MRI and WB-DW-MRI as compared with FDG-PET/CT in lymphoma patients.

The aim of this study was therefore to prospectively evaluate the evaluate the diagnostic performance of FDG-PET/MRI and WB-DW-MRI compared with FDGFDG-PET/CT using a tri-modality PET/CT-MRI system that allows for a one-stop examination in a realistic everyday clinical setting including pretreatment staging, interim and end of treatment restaging, and surveillance.

\section{Methods}

\section{Patient population}

From April 2012 through January 2014, all patients referred for a clinical FDG-PET/CT examination for either staging or restaging lymphoma were offered an additional scientific MRI within a tri-m

odality setup. A total of 82 examinations were performed in 61 patients, with 15 patients undergoing more than one scan (ten patients, two examinations; four patients, three examinations; and one patient, four examinations). No further patient inclusion criteria were applied. Exclusion criteria were unwillingness to participate in the study, claustrophobia, MRI-incompatible medical devices (e.g., cardiac pacemakers, neurostimulators, cochlear implants, and insulin pumps), or possible presence of metallic fragments in the body. This prospective study was approved by the ethics committee of the Canton of Zurich and signed informed consent was obtained from all patients prior to the examinations.

\section{FDG-PET/CT and MRI}

Sequential FDG-PET/CT and MRI were performed on a tri-modality PET/CT-MRI setup (full ring, time-of-flight Discovery PET/CT 690, 3 T Discovery MR 750w, both GE Healthcare, Waukesha, WI, USA). The dedicated MRIand CT-compatible shuttle transfer mechanism connecting the MRI and PET/CT systems allowed for PET/CT scanning free of radiofrequency (RF) coil-induced artifacts and ascertained the placement of dedicated RF coils for MRI without repositioning of the patient [8, 9].

Patients fasted for at least $4 \mathrm{~h}$ prior to injection of a standard FDG dose of $4.5 \mathrm{MBq}$ per $\mathrm{kg}$ body weight [10]. After an uptake time of $30 \mathrm{~min}$ the patient was positioned on the shuttle table in the MRI suite and MRI acquisition covering the region from the head to the upper thighs was started. The images were acquired by use of a GEM whole-body suite (GE Healthcare, Waukesha, WI, USA). The MRI protocol included a T1-weighted three-dimensional spoiled gradient echo pulse sequence (LAVA) and diffusion-weighted images obtained in the axial plane, both divided into four stations, with a total MRI scan duration of 15-20 min (see Table 1 for scanning parameters).

After completion of the MRI, coils were removed and the patients were transferred to the PET/CT, still positioned on the shuttle board. In this way, it was ensured that positioning of the patient within the PET/CT and the MRI scanners was exactly the same.

Table $1 \mathrm{MRI}$ scanning parameters

\begin{tabular}{lll}
\hline Parameter & T1w LAVA & DWI EPI-STIR \\
\hline Repetition time/echo time (ms) & $4.3 / 1.3$ & $4175 / 100$ \\
Echo train length & NA & NA \\
Flip angle $\left(^{\circ}\right.$ ) & 12 & NA \\
Inversion time (ms) & NA & 200 \\
Parallel imaging acceleration factor & 2 & 2 \\
Receiver bandwidth (kHz) & 142.86 & 250 \\
Field of view (cm) & 50 & 44 \\
Matrix & $288 \times 224$ & $64 \times 128$ \\
b value (s/mm) & NA & $0,50,500$ \\
NEX & 1 & NA \\
Number of directions & NA & 3 \\
\hline
\end{tabular}

Abbreviations: T1w LAVA T1-weighted spoiled gradient echo pulse sequence, $D W I$ diffusion-weighted imaging sequence, EPI-STIR echo planar imaging-short time inversion recovery, NEX number of excitations, NA not applicable 
After shuttle transfer to the adjacent PET/CT system (after an overall uptake time of $60 \mathrm{~min}$ ), unenhanced low-dose CT and PET emission data were acquired from the mid-thigh to the vertex of the skull. The low-dose CT was acquired during shallow breathing in the head, upper thorax, and pelvis areas and with non-forced expiration breath hold in the diaphragm and upper abdomen.

Tube voltage was $120 \mathrm{kV}$ (peak), reference tube current $12.35 \mathrm{~mA} /$ slice, automated dose modulation range 15-80 $\mathrm{mAs} / \mathrm{slice}$, collimation $64 \times 0.625 \mathrm{~mm}$, pitch $0.984: 1$, rotation time $0.5 \mathrm{~s}$, field of view (FOV) $50 \mathrm{~cm}$, and noise index $20 \%$. CT image sets were reconstructed using an iterative algorithm [Adaptive Statistical Iterative Reconstruction (ASIR), GE Healthcare].

The PET data were acquired in 3-D time of flight (TOF) mode with a scan duration of 2 min per bed position, a $23 \%$ overlap of bed positions, an axial FOV of $153 \mathrm{~mm}$, and a 700-mm-diameter FOV. The emission data were corrected for attenuation by use of the lowdose CT and iteratively reconstructed [matrix size $256 \times$ 256, VUE Point FX (3D TOF-OSEM) with 3 iterations, 18 subsets]. Images were filtered in image space using an in-plane Gaussian convolution kernel with a fullwidth at half-maximum (FWHM) of $4.0 \mathrm{~mm}$, followed by a standard axial filter with a three-slice kernel. This procedure has been used in this standard way in other studies as well [11].

\section{Image processing}

The acquired FDG-PET/CT and MRI images were transmitted to a dedicated review workstation (Advantage Workstation, Version 4.5, GE Healthcare, Milwaukee, WI, USA) that enables review of the PET, CT, and MRI images side by side or in fused/overlay mode (FDGPET/CT; FDG-PET/MRI). Due to use of the calibrated three-modality system, no software-based image registration was necessary. A previously conducted study validated the accuracy of image registration, with less than $4 \mathrm{~mm}$ lateral misalignment between CT, PET, and MRI data sets, which is similar to the intrinsic error assessed with phantom measurements [12].

\section{Image analysis}

Analysis was performed by a board-certified nuclear medicine physician and a board-certified radiologist with substantial experience in FDG-PET/CT. All images were evaluated for the presence of lymphoma manifestations according to the German Hodgkin Study Group (GHSG) protocol guidelines, including a total of 34 possible anatomic sites divided into nodal or organ involvement [3, 13-15].

Nodal involvement was considered to comprise lymphoma manifestation at any of the following sites: Waldeyer's ring, upper cervical, cervical, supraclavicular, infraclavicular, axillary, lung hilum, iliac and inguinal (right or left), upper mediastinum, lower mediastinum, liver hilum, spleen, splenic hilum, celiac, mesenteric, and para-aortic. Organ involvement was characterized as presence of a positive lesion for lymphoma in the lung (right or left), liver, pleura, skeleton, pericardium, bone marrow, or any other organ not previously described.

With regard to WB-DW-MRI, a positive lymphoma manifestation was represented by a high-signal lesion on high b-value WB-DW-MRI and a low signal on the corresponding ADC map, using a mean ADC of $1.2 \times 10^{-3}$ $\mathrm{mm}^{2} / \mathrm{s}$ as the threshold.

For assessment of lymphoma manifestation on FDGPET/CT and FDG-PET/MRI, a combination of morphologic and functional findings was used. The morphologic criteria for lymphoma manifestation were presence of a mass-like lesion, presence of enlarged lymph nodes greater than $1.0 \mathrm{~cm}$ in the short axis (and $1.5 \mathrm{~cm}$ for angular lymph nodes), cluster formation, irregular boundary of the lymph node capsule, and extracapsular lymph node spread. The functional criterion was defined as presence of an FDG-positive lesion with higher focal FDG uptake than liver activity (Deauville criteria, see below). For FDG-negative lesions, the morphologic criteria were used.

\section{Image validation and follow-up}

Imaging findings were validated by biopsy $(n=21)$, by follow-up imaging comprising CT, FDG-PET/CT, and/or FDG-PET/MRI $(n=32)$, or by clinical follow-up $(n=25)$. Due to loss to follow-up, five examinations (all negative on FDG-PET/CT) could not be further validated. Verification by biopsy was only available for one lesion per patient; however, FDG-PET/CT was then used as the reference method for comparison of the other modalities. The positivity of FDG-PET/CT and FDG-PET/MRI was based on Deauville criteria and lesions with FDG uptake higher than the liver uptake were considered positive (Deauville scores 4 and 5) [16]. The median follow-up estimated by the inverse Kaplan-Meier method was 9.1 months (range 0.0-21.3 months, median 8.7 months).

\section{Statistical analysis}

All statistical tests were performed using SPSS Statistics Version 22 (IBM, Armonk, NY, USA). Quantitative values were expressed as mean \pm standard deviation or median and range as appropriate. Comparisons of means and related metric measurements were performed using Student's t-test and the Wilcoxon signed rank test, respectively. All statistical tests were conducted two-sided and a $\mathrm{p}$ value less than 0.05 was considered to indicate statistical significance. 


\section{Results}

\section{Patient characteristics}

Sixty-two patients with a mean age of $55 \pm 20$ years (median 62; range 20-90) were prospectively included in this study. A total of 82 examinations were performed for primary staging $(n=14)$ and restaging $(n=68)$. Restaging consisted of interim examinations during ongoing therapy $(n=14)$, examination after end of treatment $(n=19)$, and surveillance $(n=35)$.

The majority of the examinations were done for assessment of Hodgkin's disease $(n=28)$ or diffuse large B-cell lymphoma $(n=26)$ (for details, see Table 2$)$. One patient who presented with suspicion for lymphoma was found to have sarcoidosis upon histologic verification and was later excluded, leaving 61 patients for lymphoma analysis.

\section{Detectability rate}

Overall, 188 lesions were considered positive in 29 examinations in 27 patients (see Table 3). Another 53 examinations in 34 patients were considered negative for lymphoma.

FDG-PET/MRI accurately detected 188 lesions, yielding a sensitivity of $100 \%$ compared with FDG-PET/CT. On the other hand, WB-DW-MRI detected 524 lesions, of which 125 (66.5\% of 188) lesions were true positive and 319 false positive findings. WB-DW-MRI accordingly missed 63 true positive $(33.5 \%$ of 188$)$ lesions.

\section{Detection of nodal vs. extranodal disease}

Of the 188 lesions positive for lymphoma, 170 represented nodal disease while 18 were found in extranodal sites. The distribution of FDG-positive lymphoma manifestations according to localization is shown in Table 4.

Table 2 Patient characteristics

\begin{tabular}{lll}
\hline Lymphoma entity & Examinations & Patients \\
\hline Hodgkin's disease & 28 & 21 \\
DLBCL & 26 & 18 \\
CLL & 2 & 2 \\
Follicular lymphoma & 5 & 5 \\
MALT & 1 & 1 \\
Mantle cell lymphoma & 11 & 6 \\
Marginal cell lymphoma & 2 & 2 \\
Peripheral T-cell lymphoma & 2 & 2 \\
Peripheral B-cell lymphoma & 1 & 1 \\
Large cell lymphoma & 1 & 1 \\
Angioblastic lymphoma & 2 & 1 \\
Sarcoidosis (excluded) & 1 & 1 \\
Mycosis fungoides & 1 & 61 \\
Total included & 82 & 1
\end{tabular}

Abbreviations: DLBCL diffuse large B-cell lymphoma, CLL chronic lymphocytic leukemia, MALT mucosa-associated lymphoid tissue
Table 3 Clinical consensus in respect of Ann Arbor stage

\begin{tabular}{lllll}
\hline & Stage I & Stage II & Stage III & Stage IV \\
\hline All & 6 & 8 & 8 & 7 \\
Primary staging & 3 & 2 & 6 & 2 \\
Interim scan & 1 & 1 & 1 & 1 \\
End of Tx & 1 & 2 & 0 & 1 \\
Surveillance & 1 & 3 & 1 & 3 \\
\hline
\end{tabular}

FDG-PET/MRI detected all 170 instances of nodal disease and also identified all 18 extranodal lymphoma manifestations; by comparison, WB-DW-MRI characterized $115(67.6 \%)$ and $10(55.6 \%)$ lesions as positive for nodal and extranodal disease, respectively (Fig. 1). Among the extranodal manifestations, splenic involvement was the source of the greatest discrepancy, with WB-DW-MRI detecting only $50 \%$ of cases and yielding false positive findings in three other patients (Fig. 2).

Table 4 Lymphoma manifestations according to the German Hodgkin Study Group (GHSG) protocol guidelines $(n=188)$

\begin{tabular}{|c|c|}
\hline Region & $N$ \\
\hline Waldeyer left & 3 \\
\hline Waldeyer right & 4 \\
\hline Upper cervical left & 9 \\
\hline Upper cervical right & 9 \\
\hline Cervical left & 11 \\
\hline Cervical right & 10 \\
\hline Supraclavicular left & 11 \\
\hline Supraclavicular right & 10 \\
\hline Infraclavicular left & 9 \\
\hline Infraclavicular right & 7 \\
\hline Axillary left & 7 \\
\hline Axillary right & 8 \\
\hline Upper mediastinum & 12 \\
\hline Lower mediastinum & 10 \\
\hline Lung hilum left & 2 \\
\hline Lung hilum right & 5 \\
\hline Spleen & 6 \\
\hline Splenic hilum & 1 \\
\hline Liver hilum & 4 \\
\hline Celiac & 4 \\
\hline Para-aortic & 6 \\
\hline Mesenteric & 6 \\
\hline Iliac left & 4 \\
\hline Iliac right & 9 \\
\hline Inguinal left & 3 \\
\hline Inguinal right & $\varepsilon$ \\
\hline Organ & 10 \\
\hline
\end{tabular}




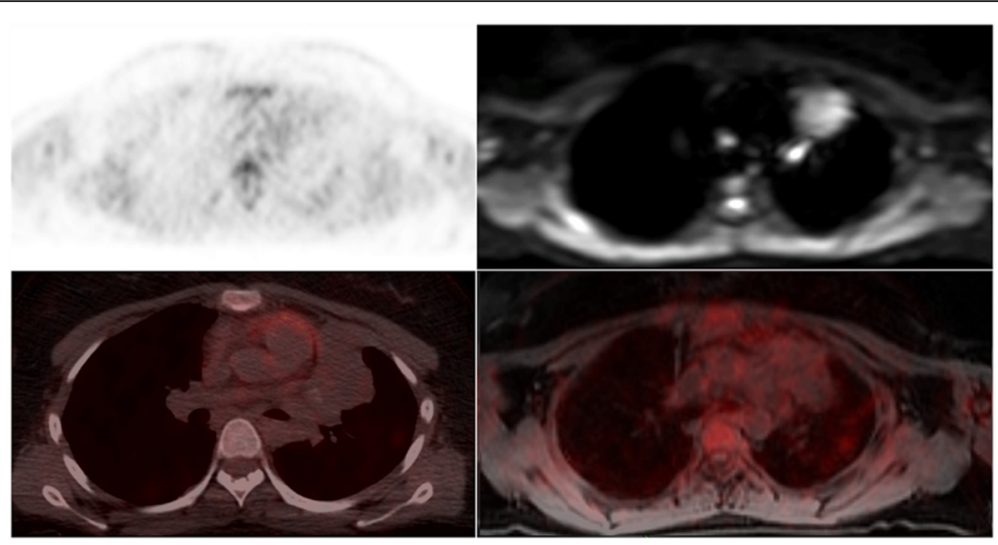

Fig. 1 A male patient with Hodgkin's disease stage IIIE. PET/CT/MRI after two cycles of chemotherapy. Top: Axial PET shows very faint uptake in the anterior mediastinal lesion; axial WB-DW-MRI $(b$ value $=800)$ shows restricted diffusion (calculated $\left.A D C_{\text {mean }}=0.96 \times 10^{-3} \mathrm{~mm}^{2} / \mathrm{s}\right) . B$ ottom: FDG-PET/CT and FDG-PET/MRI show a residual mediastinal mass without significant FDG activity. FDG-PET/CT and FDG-PET/MRI after the end of treatment confirmed complete response

\section{Staging vs. restaging}

Among the 188 lesions positive for lymphoma, 113 (60.1\%) were found in patients included for primary staging and 75 (39.9\%) in those included for restaging. Among the primary staging patients, FDG-PET/MRI accurately detected all positive lesions while WB-DW-MRI identified 73 (64.6\%) lesions. Among the patients undergoing restaging, FDG-PET/MRI and WB-DW-MRI characterized $75(100 \%)$ and $52(69.3 \%)$ lesions, respectively.

\section{Interim vs. end of treatment vs. surveillance}

FDG-PET/CT and FDG-PET/MRI detected the same number of lesions in patients who underwent examination during ongoing therapy $(n=16)$, after the end of treatment $(n=12)$, and during surveillance $(n=47)$, while WB-DW-MRI detected nine (56.3\%), six (50.0\%), and 37 (78.7 \%) lesions, respectively.

\section{Hodgkin's disease (HD) vs. diffuse large B-cell lymphoma (DLBCL) and low- and intermediate- vs. high-grade lymphoma}

Of the 82 examinations included, 28 were indicated for HD and 26 for DLBCL, accounting for a total number of 66 and 61 of the detected lesions, respectively. WB-DWMRI accurately detected 40 lesions (60.6\%) in HD patients and 41 DLBC patients $(67.2 \%)$. Fifty-four examinations were performed for evaluation of high-grade lymphomas, with FDG-PET/MRI detecting 127 positive lesions and WB-DW-MRI, 81 (63.8\%). The remaining 28 examinations were performed for evaluation of low-

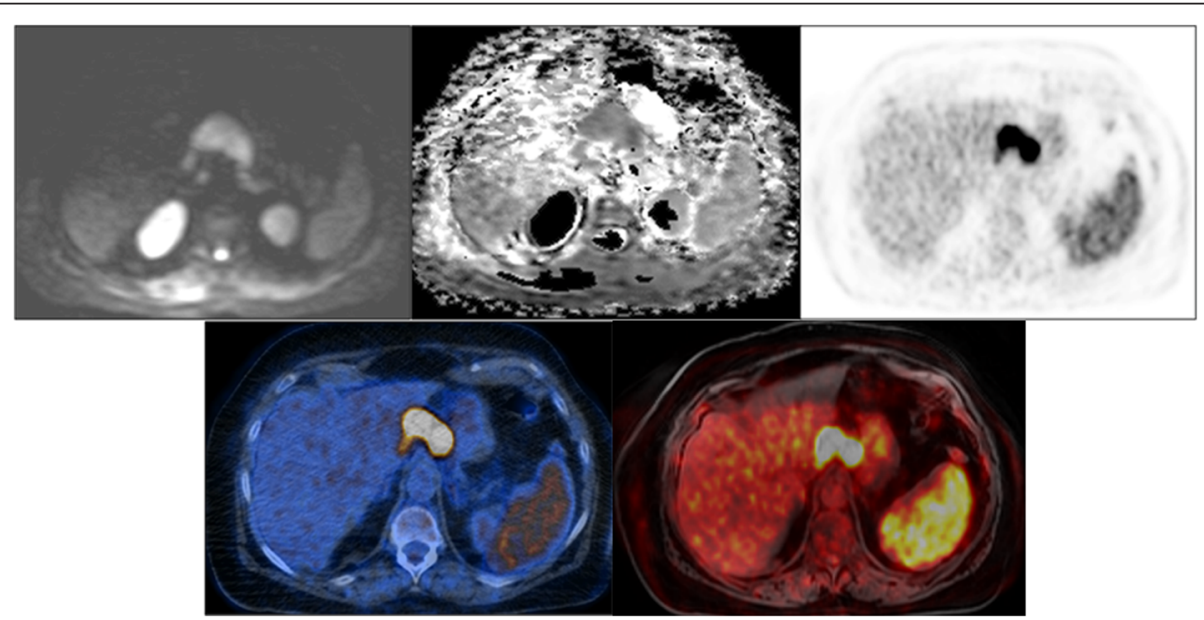

Fig. 2 A female patient with a diffuse large B-cell lymphoma stage IVB. PET/CT/MRI for initial staging. Top: Axial WB-DW-MRI $(b$ value $=800)$ and axial $A D C$ map show restricted diffusion in a lymph node conglomerate in the upper abdomen (calculated $A D C_{\text {mean }}=0.72 \times 10^{-3} \mathrm{~mm}^{2} / \mathrm{s}$ ), $\mathrm{but}_{\mathrm{n}} \mathrm{no}$ restricted diffusion in the spleen (calculated $A D C_{\text {mean }}=1.37 \times 10^{-3} \mathrm{~mm}^{2} / \mathrm{s}$ ). Axial PET shows uptake in the same lymph node conglomerate but also diffuse uptake in the spleen, which was significantly higher than liver uptake. Bottom: FDG-PET/CT and FDG-PET/MRI show FDG avidity in both the lymph node mass and the spleen, indicating lymphoma manifestation 
and intermediate-grade lymphomas. Here, all 61 lesions considered positive for lymphoma were accurately detected by FDG-PET/MRI, while 46 (75.4 \%) were detected with WB-DW-MRI.

\section{Ann Arbor stage}

In 18 examinations, WB-DW-MRI and FDG-PET/MRI agreed with respect to Ann Arbor stage (8 stage 0, 1 stage I, 0 stage II, 4 stage III, 1 stage IIIS, and 4 stage IV). Among the other 64 examinations, WB-DW-MRI resulted in upstaging in 60 cases, including 45 patients who were free of disease as determined by FDG-PET/ CT (WB-DW-MRI changed the stage from 0 to I in 9 patients, 0 to II in 10 patients, 0 to III in 25 patients, and 0 to IV in 1 patient), and downstaging in four (from IIIS to III in 1 patient and from IV to III in 3 patients). Among the 27 patients with positive findings for lymphoma, WB-DW-MRI and FDG-PET/MRI agreed in ten patients (34.5\%) while upstaging was observed in 15 $(51.7 \%)$ and downstaging in four (13.8\%).

A summary of the comparative results for FDG-PET/ CT, FDG-PET/MRI, and WB-DW-MRI is provided in Table 5.

\section{Discussion}

The results of this study show that the diagnostic performance of FDG-PET/MRI in lymphoma patients in a realistic everyday clinical setting is equal to that of FDG$\mathrm{PET} / \mathrm{CT}$, which is nowadays widely accepted as the modality of choice for staging and restaging of lymphoma patients. On the other hand, the performance of WB-DWMRI seems to be inferior to that of FDG-PET/CT/MRI in various respects, most notably for staging, differentiation of nodal and extranodal disease, and differentiation of

Table 5 Comparison of number of positive lymphoma lesions detected by FDG-PET/CT, FDG-PET/MRI, and WB-DW-MRI

\begin{tabular}{|c|c|c|c|c|}
\hline & $\begin{array}{l}\mathrm{PET} / \mathrm{CT}=\mathrm{PET} / \\
\mathrm{MRI}\end{array}$ & $\begin{array}{l}\text { WB-DW- } \\
\text { MRI }\end{array}$ & $\begin{array}{l}\text { Detectability rate } \\
\text { with WB-DW-MRI }\end{array}$ & $P$ value \\
\hline Lesions detected & 188 & 125 & $66.5 \%$ & $<0.001$ \\
\hline Nodal disease & 170 & 115 & $67.6 \%$ & $<0.001$ \\
\hline Extranodal disease & 18 & 10 & $55.6 \%$ & 0.004 \\
\hline Staging & 113 & 73 & $64.6 \%$ & 0.008 \\
\hline Restaging & 75 & 52 & $69.3 \%$ & 0.010 \\
\hline Interim & 16 & 9 & $56.3 \%$ & 0.169 \\
\hline End of treatment & 12 & 6 & $50.0 \%$ & 0.083 \\
\hline Surveillance & 47 & 37 & $78.7 \%$ & 0.058 \\
\hline High-grade & 127 & 81 & $63.8 \%$ & 0.003 \\
\hline Low-grade & 61 & 46 & $75.4 \%$ & 0.032 \\
\hline $\mathrm{HD}$ & 66 & 40 & $60.6 \%$ & 0.006 \\
\hline DLBCL & 61 & 41 & $67.2 \%$ & 0.009 \\
\hline
\end{tabular}

Abbreviations: HD Hodgkin's disease, DLBCL diffuse large B-cell lymphoma high-grade and low-grade lymphoma. The performance of WB-DW-MRI was better for evaluation during surveillance and in the assessment of low-grade lymphomas (cf. Table 5).

FDG-PET/CT and FDG-PET/MRI showed agreement for all lesions, which is not too surprising given that the PET component was the same. Differences between MRI and CT have been especially described for detection of bone marrow (MRI superior) [17] and lung involvement [3] (CT superior). However, in our study population, in which lung involvement was present in only three patients and bone marrow involvement in only one patient, FDG-PET/CT and FDG-PET/MRI were in agreement due to the increased FDG uptake in all corresponding lesions.

The equivalent performance of FDG-PET/CT and FDG-PET/MRI in patients with lymphoma was recently confirmed in a retrospective study including 33 patients and a total of 702 lymph node stations [18]. Using FDG$\mathrm{PET} / \mathrm{CT}$ as the reference standard, FDG-PET/MRI had a sensitivity of $93.8 \%$ and a specificity of $99.4 \%$, results which are in line with those of our study.

For WB-DW-MRI, ADC values were determined for any lesions visually detectable, as no definite cut-offs have previously been reported. We evaluated the entire data set using different cut-offs for the ADC (data not shown), and the cut-off selected (mean ADC threshold of $1.2 \times 10-3 \mathrm{~mm} 2 / \mathrm{s}$ ) performed best in terms of overall accuracy. Nevertheless, the difficulty in identification of a cut-off explains the very high number of false positive lesions on WB-DW-MRI, which in general detected two-thirds of lymphoma lesions. Difficulty in deriving an optimal cut-off for the ADC value was also reported by Punwani et al. in 39 patients undergoing WB-DW-MRI and FDG-PET/CT before and after two cycles of chemotherapy. Interim $\mathrm{ADC}$ values in patients with adequate FDG-PET/CT response were not statistically different from those in patients without an adequate response [7]. PET imaging detects lymphoma activity on the basis of tumor glucose metabolism, while WB-DW-MRI does so on the basis of the motion of water molecules in a densely cellular environment. Our findings show - as do those of several previous publications - that tumor cellularity as detected by WB-DW-MRI may not be an adequate marker for lymphoma activity to the same extent as glycolytic metabolism. This inference is supported by the findings of $\mathrm{Wu}$ et al., who concluded, on the basis of results in patients with histologically proven DLBCL, that SUV for PET and ADC for WB-DW-MRI are different indices for the characterization of lymphomas [19].

When our patient population was categorized into different subsets according to lymphoma manifestation (nodal vs. extranodal disease), indication (staging vs. 
restaging), timepoint of examination (during ongoing therapy, after end of treatment, and surveillance) and grade of lymphoma (low- vs. high-grade and HD vs. DLBCL), WB-DW-MRI failed to achieve the lesion detectability offered by FDG-PET/CT or FDG-PET/MRI in any of the subsets. Kwee et al. recently reported that WB-DW-MRI did not provide any advantage over MRI without DWI in 108 newly diagnosed lymphoma patients [3]. Slightly improved results were reported by Tsuji et al., who compared FDG-PET/CT and MRI in 28 malignant lymphoma patients prior to any treatment and after two cycles of chemotherapy [20]. While concordant findings were reported in 22/28 (79 \%) patients, significant differences were nevertheless found between FDG-PET/CT and MRI. The best results were obtained in the study of Mayerhoefer and co-workers, who reported WB-DW-MRI to have a region-based sensitivity of $97 \%$ compared with FDG-PET/CT in known FDGavid lymphoma histologic subtypes [21].

In our study, the results of WB-DW-MRI were not statistically different from those of the reference standard, FDG-PET/CT, with respect to interim/end of treatment imaging and surveillance. These findings are to an extent similar to the results of Mayerhoefer and co-workers in lymphomas with variable FDG avidity [21]. However, the impact of the low numbers of lesions in the relevant subsets in our study has to be borne in mind.

As WB-DW-MRI achieved an almost comparable detection rate to FDG-PET/CT among patients undergoing surveillance, this method might be considered for follow-up of this subgroup of patients when baseline imaging with WB-DW-MRI is available, especially given that FDG-PET/CT in general is not recommended for this purpose [1].

WB-DW-MRI showed inferior results in the evaluation of extranodal disease and for overall restaging. Only a few studies have evaluated the accuracy of WBDW-MRI and FDG-PET/MRI for detection of extranodal disease. Results of other studies have suggested that, overall, WB-DW-MRI and FDG-PET/MRI may have an advantage compared with FDG-PET/CT for this purpose $[22,23]$, especially when considering bone marrow involvement. In our study, only one patient presented bone marrow infiltration, so we cannot offer further comment on this aspect. However, we did find that diffuse splenic involvement may not be reliably detected by WB-DW-MRI; this confirms previous observations by Toledano-Massiah and colleagues [22] and reflects the fact that restricted diffusion may be observed even in a normal spleen.

For therapy response assessment, FDG-PET/MRI has proved to be feasible and reliable [24]. Most studies describe an elevation in the ADC mean value as suggestive of response to treatment [25-27]. Our study has shown that, when used for restaging, WB-DW-MRI performed less well than FDG-PET/MRI in detecting lymphoma activity. This finding suggests that the use of WB-DWMRI to assess treatment response of lymphoma may underestimate the true number of lesions and that careful evaluation is required in order to avoid false negative findings.

We observed only moderate agreement between WBDW-MRI and FDG-PET/MRI or FDG-PET/CT concerning determination of the Ann Arbor stage. One of the reasons for this may be the lack of standardized criteria for definition of lymphoma involvement on WB-DWMRI, which may be considered responsible for the very high number of false positive lesions in our study. As indicated above, we tested our results with different ADC thresholds (data not shown). When the threshold was changed, however, the values for sensitivity and specificity altered in opposite directions (e.g., sensitivity increased but specificity decreased) and no improvement in overall accuracy was achieved. Another drawback is that no parameters have been defined for the evaluation of extranodal disease. Hence, the reproducibility of WBDW-MRI is limited and may also be partly dependent on the MRI scanner used.

Overall, our results indicate that the similarity in diagnostic performance of FDG-PET/CT and FDG-PET/MRI reported previously in various solid tumors also holds true for FDG-avid lymphoma types. While the findings of most studies cited above are generally in line with our own results, the reported inferiority of WB-DW-MRI compared with FDG-PET-based techniques is somewhat at odds with a few other studies in the literature. One potential explanation is our choice of everyday setting including a wide range of histologies and different clinical situations ranging from staging to surveillance, as well as the use of a tri-modality system with MRI being performed separately from the PET component. Moreover, it is well known that bone marrow, spleen, and lymph nodes retain high signal intensity and are therefore difficult to assess with WB-DW-MRI [28].

When interpreting the results of this study, several limitations have to be taken into account. First, histopathology as the reference standard of choice was not available in all lesions (ethically this was not possible), though it was usually available in patients referred for initial staging. However, FDG-PET/CT is widely accepted as a reference standard to determine disease in lymphoma [1]. Additionally, our study did not define a threshold for lesion size in WB-DW-MRI and consequently, we detected a very high number of false positive lesions, resulting in overestimated upstaging. However, even without such a threshold, WB-DW-MRI was unable to detect all of the lesions that were positive on FDG-PET. Finally, we used a tri-modality PET/CT-MRI setup rather than 
simultaneous PET//MRI and thus the attenuation correction for the PET component was always based on the lowdose CT.

\section{Conclusion}

In summary, our results indicate that FDG-PET/CT and FDG-PET/MRI have a similar performance in the clinical work-up of lymphomas, while WB-DW-MRI is inferior to both FDG-PET-based methods. WB-DW-MRI can, however, be used in specific scenarios, e.g., in low-grade lymphomas as well as imaging during surveillance.

\section{Abbreviations}

WB-DW-MRI: Whole-body diffusion-weighted magnetic resonance imaging; MRI: Magnetic resonance imaging; PET: Positron emission tomography; FDG: Fluorodeoxyglucose; CT: Computed tomography; ADC: Apparent diffusion coefficient; CT: Computed tomography; RF: Radiofrequency; MBq: Megabecquerel; GEM: Geometry embracing method; FOV: Field of view; GHSG: German Hodgkin Study Group; DLBCL: Diffuse large B-cell lymphoma; CLL: Chronic lymphocytic leukemia; MALT: Mucosa-associated lymphoid tissue.

\section{Competing interests}

Patrick Veit-Haibach received IIS grants from Bayer Healthcare, Siemens Healthcare, Roche Pharmaceuticals, and GE Healthcare and speaker fees from GE Healthcare. For the remaining authors none were declared.

\section{Authors' contributions}

$\mathrm{KH}$ made substantial contributions to analysis and interpretation of data, drafted the manuscript, and performed the statistical analysis. MQ made substantial contributions to analysis and interpretation of data and revised the manuscript critically for important intellectual content. $\mathrm{MH}, \mathrm{AB}, \mathrm{NS}$, and PS revised the manuscript critically for important intellectual content. FGB prepared the figures and revised the manuscript critically for important intellectual content. PVH participated in its design and coordination and revised the manuscript critically for important intellectual content. All authors read and approved the final manuscript.

\section{Acknowledgments}

The authors thank the personnel at the Department of Nuclear Medicine of University Hospital of Zurich for technical support.

\section{Author details}

'Department of Nuclear Medicine, University Hospital Zurich, Rämistrasse 100, CH-8091 Zurich, Switzerland. 'Department of Nuclear Medicine, Universitätsklinikum Würzburg, Oberdürrbacher Str. 6, DE-97080 Würzburg, Germany. ${ }^{3}$ Department of Neuroradiology, University Hospital Zurich, Rämistrasse 100, CH-8091 Zurich, Switzerland. ${ }^{4}$ Department of Diagnostic and Interventional Radiology, University Hospital Zurich, Rämistrasse 100, CH-8091 Zurich, Switzerland. ${ }^{5}$ Department of Medical Oncology, University Hospital Zurich, Rämistrasse 100, CH-8091 Zurich, Switzerland. ${ }^{6}$ University of Zurich, Zurich, Switzerland.

Received: 21 August 2015 Accepted: 15 December 2015 Published online: 23 December 2015

\section{References}

1. Cheson BD, Fisher Rl, Barrington SF, Cavalli F, Schwartz LH, Zucca E, et al. Recommendations for initial evaluation, staging, and response assessment of Hodgkin and Non-Hodgkin lymphoma: the Lugano classification. J Clin Oncol. 2014;32:3059-68.

2. Koh D-M, Collins DJ. Diffusion-weighted MRI in the body: applications and challenges in oncology. Am J Roentgenol. 2007;188:1622-35.

3. Kwee TC, Vermoolen MA, Akkerman EA, Kersten MJ, Fijnheer R, Ludwig I, et al. Whole-body MRI, including diffusion-weighted imaging, for staging lymphoma: comparison with CT in a prospective multicenter study. J Magn Reson Imaging. 2014;40:26-36.
4. Lin C, Luciani A, Itti E, El-Gnaoui T, Vignaud A, Beaussart P, et al. Whole-body diffusion-weighted magnetic resonance imaging with apparent diffusion coefficient mapping for staging patients with diffuse large B-cell lymphoma. Eur Radiol. 2010:20:2027-38.

5. Van Ufford HMEQ, Kwee TC, Beek FJ, van Leeuwen MS, Takahara T, Fijnheer R, et al. Newly diagnosed lymphoma: initial results with whole-body T1-weighted, STIR, and diffusion-weighted MRI compared with 18F-FDG PET/CT. AJR Am J Roentgenol. 2011;196:662-9.

6. Bailey DL, Barthel H, Beyer T, Boellaard R, Gückel B, Hellwig D, et al. Summary report of the First International Workshop on PET/MR imaging, March 19-23, 2012, Tübingen, Germany. Mol Imaging Biol. 2013;15:361-71.

7. Punwani S, Taylor SA, Saad ZZ, Bainbridge A, Groves A, Daw S, et al. Diffusion-weighted MRI of lymphoma: prognostic utility and implications for PET/MRI? Eur J Nucl Med Mol Imaging. 2013;40:373-85.

8. Kuhn FP, Crook DW, Mader CE, Appenzeller P, von Schulthess GK, Schmid DT. Discrimination and anatomical mapping of PET-positive lesions: comparison of $\mathrm{CT}$ attenuation-corrected PET images with coregistered MR and CT images in the abdomen. Eur J Nucl Med Mol Imaging. 2012;40(1): 44-51. doi:10.1007/s00259-012-2236-3.

9. Veit-Haibach P, Kuhn FP, Wiesinger F, Delso G, von Schulthess G. PET-MR imaging using a tri-modality PET/CT-MR system with a dedicated shuttle in clinical routine. MAGMA. 2013;26:25-35.

10. Boellaard R, Delgado-Bolton R, Oyen WJG, Giammarile F, Tatsch K, Eschner W, et al. FDG PET/CT: EANM procedure guidelines for tumour imaging: version 2.0. Eur J Nucl Med Mol Imaging. 2014;42(2):328-54. doi:10.1007/s00259-014-2961-X.

11. Appenzeller P, Mader C, Huellner MW, Schmidt D, Schmid D, Boss A, et al. PET/CT versus body coil PET/MRl: how low can you go? Insights Imaging. 2013;4:481-90.

12. Samarin A, Kuhn FP, Brandsberg F, von Schulthess G, Burger IA. Image registration accuracy of an in-house developed patient transport system for PET/CT + MR and SPECT + CT imaging. Nucl Med Commun. 2015;36:194-200.

13. Perrone A, Guerrisi P, Izzo L, D'Angeli I, Sassi S, Lo ML, et al. Diffusionweighted MRI in cervical lymph nodes: differentiation between benign and malignant lesions. Eur J Radiol. 2011;77:281-6.

14. Wu X, Pertovaara H, Dastidar P, Vornanen M, Paavolainen L, Marjomäki V, et al. ADC measurements in diffuse large B-cell lymphoma and follicular Iymphoma: a DWI and cellularity study. Eur J Radiol. 2013;82:e158-64.

15. Wu X, Pertovaara H, Korkola P, Dastidar P, Järvenpää R, Eskola H, et al. Correlations between functional imaging markers derived from PET/CT and diffusion-weighted MRI in diffuse large B-cell lymphoma and follicular lymphoma. PLoS One. 2014;9:e84999.

16. Meignan M, Gallamini A, Haioun C. Report on the first international workshop on interim-PET-scan in lymphoma. Leuk Lymphoma. 2009;50:1257-60.

17. Kwee TC, Kwee RM, Verdonck LF, Bierings MB, Nievelstein RAJ. Magnetic resonance imaging for the detection of bone marrow involvement in malignant lymphoma. Br J Haematol. 2008;141:60-8.

18. Platzek I, Beuthien-Baumann B, Ordemann R, Maus J, Schramm G, Kitzler HH, et al. FDG PET/MR for the assessment of lymph node involvement in lymphoma. Initial results and role of diffusion-weighted MR. Acad Radiol. 2014;21(10):1314-9. doi:10.1016/j.acra.2014.05.019.

19. Wu X, Korkola P, Pertovaara $H$, Eskola $H$, Järvenpää R, Kellokumpu-Lehtinen P-L. No correlation between glucose metabolism and apparent diffusion coefficient in diffuse large B-cell lymphoma: a PET/CT and DW-MRI study. Eur J Radiol. 2011;79:e117-21.

20. Tsuji K, Kishi S, Tsuchida T, Yamauchi T, Ikegaya S, Urasaki Y, et al. Evaluation of staging and early response to chemotherapy with whole-body diffusionweighted MRI in malignant lymphoma patients: A comparison with FDGPET/CT. J Magn Reson Imaging. 2015;41:1601-7.

21. Mayerhoefer ME, Karanikas G, Kletter K, Prosch H, Kiesewetter B, Skrabs C, et al. Evaluation of diffusion-weighted MRI for pretherapeutic assessment and staging of lymphoma: results of a prospective study in 140 patients. Clin Cancer Res. 2014;20:2984-93.

22. Toledano-Massiah S, Luciani A, Itti E, Zerbib P, Vignaud A, Belhadj K, et al. Whole-body diffusion-weighted imaging in Hodgkin lymphoma and diffuse large B-cell lymphoma. Radiographics. 2015;35:747-64.

23. Heacock L, Weissbrot J, Raad R, Campbell N, Friedman KP, Ponzo F, et al. PET/MRI for the evaluation of patients with lymphoma: initial observations. Am J Roentgenol. 2015;204(April):842-8.

24. Platzek I, Beuthien-Baumann B, Langner J, Popp M, Schramm G, Ordemann $R$, et al. PET/MR for therapy response evaluation in malignant lymphoma: initial experience. Magn Reson Mater Physics Biol Med. 2013;26:49-55. 
25. Wu X, Nerisho S, Dastidar $P$, Ryymin $P$, Järvenpää $R$, Pertovaara $H$, et al Comparison of different MRI sequences in lesion detection and early response evaluation of diffuse large B-cell lymphoma-a whole-body MRI and diffusion-weighted imaging study. NMR Biomed. 2013;26:1186-94

26. Lin C, Itti E, Luciani A, Zegai B, Lin S, Kuhnowski F, et al. Whole-body diffusion-weighted imaging with apparent diffusion coefficient mapping for treatment response assessment in patients with diffuse large B-cell lymphoma: pilot study. Invest Radiol. 2011;46:341-9.

27. Chen Y, Zhong J, Wu H, Chen N. The clinical application of whole-body diffusion-weighted imaging in the early assessment of chemotherapeutic effects in lymphoma: the initial experience. Magn Reson Imaging. 2012;30:165-70.

28. Kwee TC. Can whole-body MRI replace (18)F-fluorodeoxyglucose PET/CT? Lancet Oncol. 2014;15:243-4.

Submit your next manuscript to BioMed Central and we will help you at every step:

- We accept pre-submission inquiries

- Our selector tool helps you to find the most relevant journal

- We provide round the clock customer support

- Convenient online submission

- Thorough peer review

- Inclusion in PubMed and all major indexing services

- Maximum visibility for your research

Submit your manuscript at www.biomedcentral.com/submit
Biomed Central 